\title{
Lichtabsorption durch freie Ladungsträger in Metalloxiden: Anwendung der Brechungsindex-Modulation in der Gassensorik
}

\author{
Xia W $u^{1}$, Xuyang Zhang ${ }^{1}$, Sabrina Amrehn ${ }^{1}$, Thorsten Wagner ${ }^{1}$ \\ ${ }^{1}$ Department Chemie, Universität Paderborn, Warburger Straße 100, 33098 Paderborn
}

\section{Zusammenfassung}

Einige halbleitende Metalloxide, wie z.B. Wolframtrioxid $\left(\mathrm{WO}_{3}\right)$, zeigen gasinduzierte Änderungen ihrer elektronischen Eigenschaften und finden deshalb Anwendung als Wirkschichten in resistiven Halbleitergassensoren. Den mit den elektronischen Änderungen korrelierten, gasinduzierten optischen Eigenschaftsänderungen wurde bisher jedoch wenig Aufmerksamkeit gewidmet. Die verfügbaren Studien zeigen oft nur schwache optische Gasreaktionen in den beobachteten Wellenlängenbereichen, obwohl starke elektrische Signale zu erwarteten wären. Die vorliegende Arbeit soll mit Hilfe von Wolframoxid als Modellmaterial helfen, diese offensichtliche Diskrepanz aufzuklären. Mit einer Reihe von photonischen Kristallen wird die wasserstoffinduzierte Dispersion des Brechungsindexes des $\mathrm{WO}_{3}$ bzw. einer Wolframbronze durch Analyse der jeweiligen optischen Reaktion rekonstruiert. Es zeigt sich, dass die gasinduzierte Absorption von freien Ladungsträgern der dominierende Mechanismus für die Modulation des Brechungsindexes ist. Diese Modulation ist im nahen Infrarot stark, im sichtbaren Bereich jedoch schwach, was die bisher beobachteten, relativ geringen optischen Reaktionen erklärt. Durch die Verwendung von photonischen Kristallen mit einer optischen Bandlücke im nahen Infrarotbereich erhöht sich die optische Antwort des hier vorgestellten $\mathrm{WO}_{3} / \mathrm{H}_{2}$-Systems um eine Größenordnung. Des Weiteren wird davon ausgegangen, dass der vorgestellte Mechanismus für optische Gassensoren, die auf anderen Metalloxiden basieren, anwendbar ist. Dies ermöglicht die optische Detektion weiterer Gase mit Hilfe von Metalloxidschichten.

Keywords: Optisches Messverfahren, Gassensor, Metalloxid, chemischer Sensor, Modellbildung

\section{Einleitung}

Einige halbleitende Metalloxide zeigen gasinduzierte Änderungen der elektrischen Eigenschaften (Leitfähigkeit, Widerstand) und finden daher Anwendung als aktive Materialien in resistiven Gassensoren.

Theoretisch sollten diese Metalloxide auch optische Gasreaktionen zeigen, da die mikroskopischen elektrischen Eigenschaften der Halbleiter eng mit den makroskopischen optischen Eigenschaften verbunden sind. [1] Der Vorteil von optischen Gaswandlern besteht in der Möglichkeit der Fernauslesung. Dies bedeutet, dass kein physischer Kontakt zum Sensormaterial erforderlich ist. Dadurch kann der Anwendungsbereich von chemischen Gassensoren auf Metalloxidbasis in rauen Umgebungen, wie z.B. unter hohen Temperaturen oder in Umgebungen mit explosiven Gasen, erweitert werden.

Bisherige Studien zeigen vergleichsweise schwache optische Reaktionen von Metalloxiden, wie z.B. $\mathrm{Co}_{3} \mathrm{O}_{4}{ }^{[2]}, \mathrm{TiO}_{2}{ }^{[3]}, \mathrm{SnO}_{2}{ }^{[3]}$ oder $\mathrm{WO}_{3}{ }^{[4-7]}$, im Vergleich zu ihren elektrischen Antworten auf verschiedene Gasangebote.
Die optische Reaktion basiert in allen Fällen auf dem sogenannten gasochromen Effekt, wobei das bekannteste Beispiel die Änderung der optischen Konstanten von $\mathrm{WO}_{3}$ unter Wasserstoffatmosphäre ist. ${ }^{[4-11]}$ Für den chemischen Prozess existieren verschiedene Modellvorstellungen, die den Farbeffekten zugrunde liegen. Das weithin akzeptierte Modell im Fall des $\mathrm{WO}_{3}$ ist das sogenannte Doppelinjektionsmodell. [10,12]

$$
\mathrm{WO}_{3}+x \mathrm{e}^{-}+x \mathrm{H}^{+} \rightleftharpoons \mathrm{H}_{\mathrm{x}} \mathrm{WO}_{3}
$$

Wasserstoff wird dabei an Edelmetallkatalysatoren (z. B. Platin und Palladium) dissoziiert und die Protonen mit den begleitenden Elektronen in das $\mathrm{WO}_{3}$ injiziert; bei diesem Prozess bildet sich sogenannte Wolframbronze $\left(\mathrm{H}_{\times} \mathrm{WO}_{3}\right)$. Der daraus resultierende, weithin akzeptierte physikalische Mechanismus für den beobachteten Umfärbungseffekt im Fall von kristallinem $\mathrm{WO}_{3}$ ist die Absorption durch freie Ladungsträger; im n-Halbleiter Elektronen. 

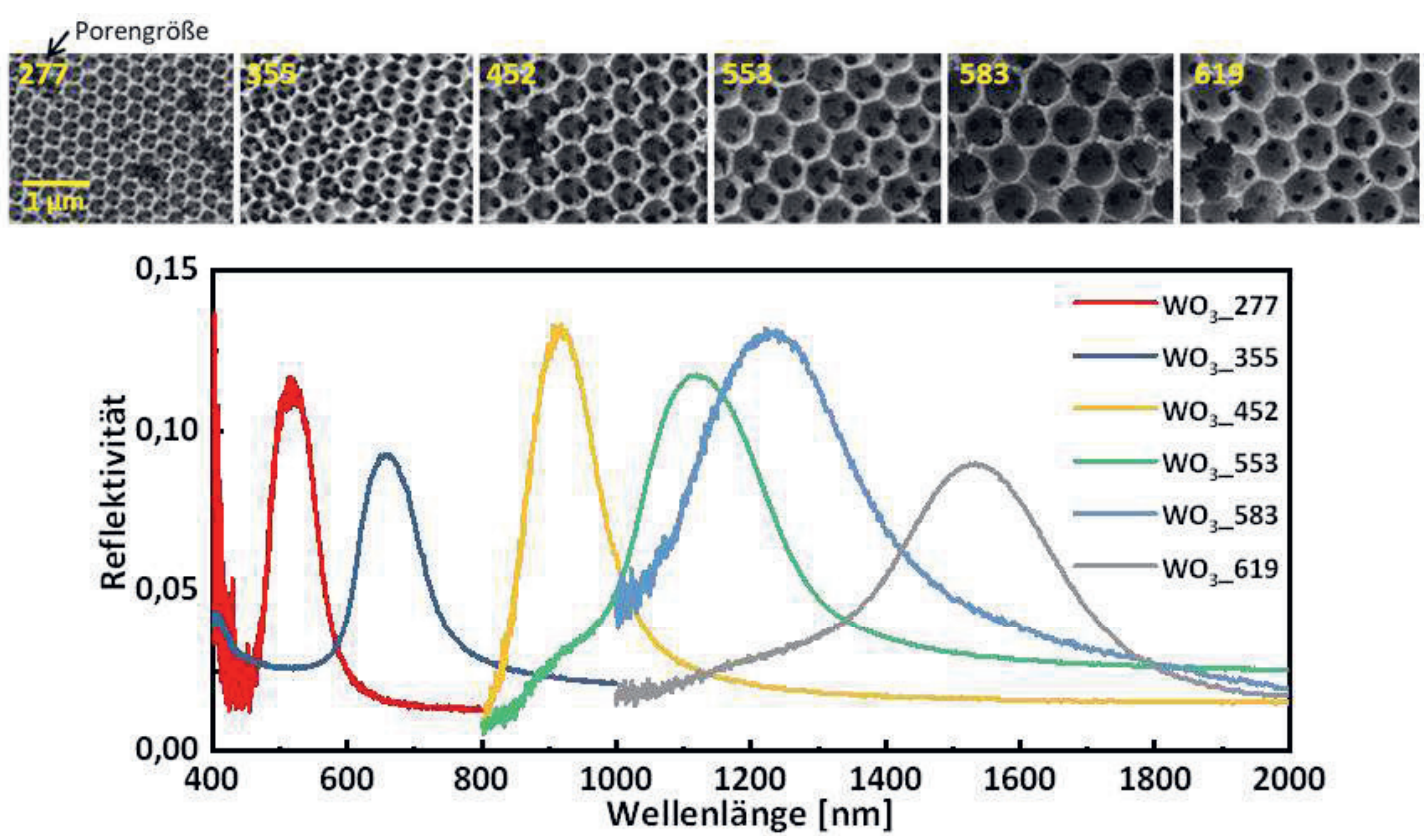

$A b b$. 1: Reflexionsspektren der verschiedenen $W_{3}$-Inversopale (unten) und elektronenmikroskopische Aufnahmen der Proben (oben); die jeweiligen Porengrößen sind in gelb angegeben.

Der freie Elektronenabsorptionseffekt ist ein Intraband-Übergang im Leitungsband, der die einfallende Photonenenergie auf die freien Elektronen überträgt, die aus dem Gas abgegeben oder durch ein elektrisches Feld injiziert werden. ${ }^{[3,4,8,12]}$ Die Absorption freier Ladungsträger ist das wichtige Bindeglied zum Verständnis der Beziehung zwischen der optischen und elektrischen Gasreaktion von Metalloxiden.

Dies ist wahrscheinlich darauf zurückzuführen, dass die freie Ladungsträgerabsorption der meisten Metalloxide im Infrarotbereich (IR) auftritt und somit für unsere Augen nicht sichtbar ist. ${ }^{[1,3]}$ Im Gegensatz dazu ist der gasochrome Effekt von $\mathrm{WO}_{3}$ nicht auf den IRBereich beschränkt, sondern tritt auch im sichtbaren Bereich auf. Aus diesem Grund betrachten wir hier die Kombination $\mathrm{WO}_{3} / \mathrm{H}_{2}$ als Modellsystem.

Wir zeigen im Folgenden, dass das tiefere mechanistische Verständnis erlaubt, die optische Antwort des $\mathrm{WO}_{3} / \mathrm{H}_{2}$-Sensorsystems um eine Größenordnung zu erhöhen. Durch geeignete Wahl der optischen Bandlücke der Inversopale kann die starke Variation des Brechungsindexes von $\mathrm{H}_{\mathrm{x}} \mathrm{WO}_{3}$ bei bestimmten Wellenlängen genutzt werden. Wichtiger ist jedoch, dass das Verständnis der optischen Auswirkung dieses Mechanismus die Verwendung weiterer Metalloxide als optische Sensormaterialien ermöglicht.

\section{Experimentelles}

Für die vorgestellte Arbeit wurden Inversopale aus $\mathrm{WO}_{3}$ mit verschiedenen Bandlücken mit Hilfe einer Abformmethode hergestellt. [13,14]

Mit Hilfe eines löslichen Metalloxid-Präkursors wurden dazu Inversopale an künstlichen Opalen aus Polymethylmethacrylat (PMMA) abgeformt. Die Größe der in der PMMAStrukturmatrix verwendeten Nanokugeln wurde im Bereich von $298 \mathrm{~nm}$ bis $644 \mathrm{~nm}$ variiert. Die so hergestellten Inversopale aus $\mathrm{WO}_{3}$ wurden anschließend mit einem Platinkatalysator beladen. Elektronenmikroskopische Aufnahmen bestätigen die gleichmäßige Porengeometrie und einen hohen Ordnungsgrad (Abb.1).

Die so erhaltenen Proben wurden mit Hilfe eines Spektrometers (FTIR, Vertex 70 mit Hyperion 1000 Mikroskop, Bruker) optisch charakterisiert (Abb.1). Im sichtbaren Bereich kam ein refraktives 4fach Objektiv (NA=0,1) zum Einsatz. Im IR-Bereich wurde ein reflektives 36fach Objektiv verwendet $(\mathrm{NA}=0,5)$. Die Gasmessungen wurden mit Hilfe eines selbstentwickelten, optischen Aufbaus durchgeführt (Abb.2). Dazu wurde Licht über eine Faser und einen Strahlteiler auf die Probe und anschließend auf das jeweilige Spektrometer geleitet. Für die Gasmessungen kamen zwei dispersive Spektrometer zum Einsatz (sichtbarer Bereich: flame, Ocean Optics; IR-Bereich: AvaSpec-USB2, Avantes). Die so erhaltenen Spektren wurden mit Hilfe 
des (Details siehe ${ }^{[13]}$ ).

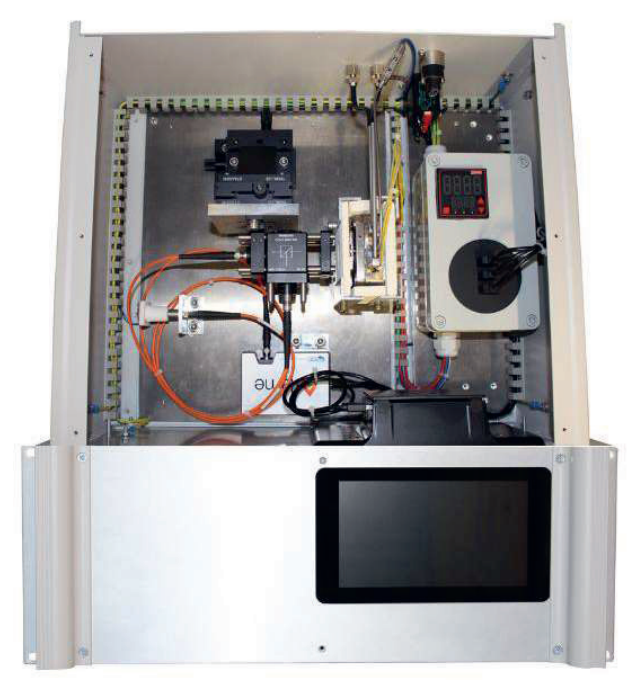

Abb. 2: Mobiler Aufbau für Gasmessungen mit Metalloxid-Inversopalen. Zentrales Element ist die Gasmesszelle mit eigebauter Heizkartusche und Fenster für optische Messungen.

Die Proben wurden auf eine Temperatur von $140^{\circ} \mathrm{C}$ aufgeheizt. Die Testgasmischungen bestanden aus $\mathrm{H}_{2}$ in trockenem Stickstoff $\left(\mathrm{N}_{2}\right)$, mit einem Konzentrationsbereich von 0,15 vol\% bis 1 vol\% $\mathrm{H}_{2}$. Nach dem jeweiligen Gasangebot wurden die Proben in synthetischer Luft regeneriert. Anschließend wurde die Messzelle vor dem nächsten Gasangebot mit trockenem Stickstoff gespült.

\section{Ergebnisse und Diskussion}

Die Abbildung (Abb. 3) zeigt exemplarisch eine Gasmessung mit dem zuvor beschriebenen Aufbau. Es ist zu erkennen, dass die Beaufschlagung mit Wasserstoff $\mathrm{zu}$ einer deutlichen Verschiebung des Reflexionsmaximums des $\mathrm{WO}_{3}$ Inversopals führt. Die Reflexionsbande vor $\mathrm{H}_{2}$-Exposition liegt bei $646 \mathrm{~nm}$ und verschiebt sich in Richtung kürzerer Wellenlängen (Blauverschiebung) unter Wasserstoffeinfluss.

Für die weitere Analyse wurden die zuvor beschriebenen Inversopale mit unterschiedlichen Bandlücken bzw. Lagen der Reflexionsbanden mit dem gleichen Messprogramm wie in Abb. 3 charakterisiert. Dadurch fungieren die Inversopale als Sonde zur Ermittlung der Dispersion der Wolframbronze $\left(\mathrm{H}_{\mathrm{x}} \mathrm{WO}_{3}\right)$ in Abhängigkeit der Wellenlänge.

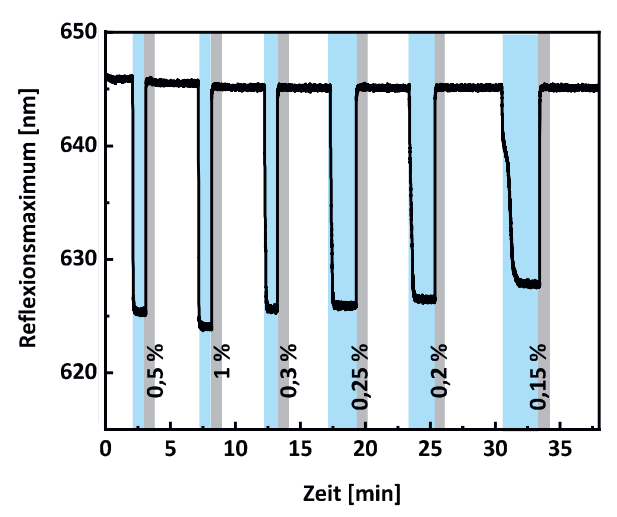

Abb. 3: Verschiebung der Reflexionsbande eines $\mathrm{WO}_{3}$ Inversopals (Porengröße: $355 \mathrm{~nm}$ ) während eine Gasmessung mit $\mathrm{H}_{2}$ bei $140{ }^{\circ} \mathrm{C}$. (blau: $\mathrm{H}_{2}$ Angebot; grau: Regenration mit synth. Luft; weiß: Spülen mit $N_{2}$ )

Mit Hilfe der optischen Sensorantwort (R), d.h. mit dem Verhältnis aus Wellenlängenverschiebung unter Gas $(\Delta \lambda)$ zur Lage der Reflexionsbande ohne Gas $\left(\lambda_{0}\right)$, wurde die Dispersion des Brechungsindexes mit folgendem Zusammenhang rekonstruiert:

$R=\Delta \lambda / \lambda_{0}=\Delta \bar{n} / \bar{n}_{0}=\left(\bar{n}_{1}-\bar{n}_{0}\right) / \bar{n}_{0}$

Der mittlere Brechungsindex des $\mathrm{WO}_{3}$ Inversopals $\left(\bar{n}_{0}\right)$ und des $\mathrm{H}_{\mathrm{xWO}} \mathrm{WO}_{3}$ Inversopals $\left(\bar{n}_{1}\right)$ können mit der allgemeinen Formel für den mittleren Brechungsindex eines photonischen Kristalls berechnet werden [15,16]:

$\bar{n}^{2}=n_{s}^{2} f_{s}+n_{p}^{2} f_{p}$,

mit $n_{s}$ und $n_{p}(=1)$ den Brechungsindizes der Festphase und der Gasphase in den Poren, und $f_{s}$ and $f_{p}\left(=1-f_{s}\right)$ den Volumenanteilen der beiden Phasen. Für den Brechungsindex des $\mathrm{WO}_{3}\left(n_{\text {oxide }}\right)$ wurde konstant 2,03 über den untersuchten Bereich angenommen. [17]

Die so rekonstruierte Dispersion ist in Abb.4 dargestellt. Die Ergebnisse zeigen, dass die stärkste Reaktion im Bereich von $965 \mathrm{~nm}$ zu finden ist. Mit Hilfe dieser Dispersionskurve ist es nun möglich, die Theorie der freien Ladungsträgerabsorption als Ursache für die beobachteten Effekte zu prüfen. Ausgehend vom sogenannten Drude-Modell:

$\epsilon(\omega)=\epsilon_{1}(\omega)+i \epsilon_{2}(\omega)=\epsilon_{\infty}-\frac{\omega_{p}^{2}}{i \omega \gamma+\omega^{2}}$

können folgende Gleichungen abgeleitet werden: 


$$
\begin{aligned}
& \epsilon_{1}(\omega)=\epsilon_{\infty}-\frac{\omega_{p}{ }^{2}}{\omega^{2}+\gamma^{2}} \\
& \epsilon_{2}(\omega)=\frac{\omega_{p}^{2} \gamma}{\omega^{3}+\omega \gamma^{2}}
\end{aligned}
$$

mit der Frequenz des Lichts $\omega$, der komplexen relative Permittivität $\epsilon(\omega)$, und dem Real- und Imaginärteilen dieser Permittivität $\epsilon_{1}(\omega)$ und $\epsilon_{2}(\omega)$. Mit Hilfe dieser Gleichungen kann der Brechungsindex der Wolframbronze $n_{\text {bronze }}(\omega)$ wie folgt beschrieben werden:

$$
n_{\text {bronze }}^{2}(\omega)=\frac{1}{2}\left(\sqrt{\epsilon_{1}^{2}+\epsilon_{2}^{2}}+\epsilon_{1}\right)
$$

Hier sind die freien Parameter für die Kurvenanpassung die Hintergrundpermittivität $\epsilon_{\infty}$ bei hohen Frequenzen, die Plasmafrequenz $\omega_{p}$, und der Dämpfungskoeffizient $\gamma$. Zentrales Ergebnis der Kurvenanpassung (Abb. 4) ist, dass der Brechungsindex der Wolframbronze $\left(n_{\text {bronze }}(\omega)\right)$ im Bereich von $500 \mathrm{~nm}$ bis $1.5 \mu \mathrm{m}$ eine starke Dispersion aufweist und die Form dem Drude-Modell gleicht. Da das DrudeModell die mikroskopische Wechselwirkung zwischen Licht und freien Ladungsträgern in einem Material beschreibt, deutet dies darauf hin, dass die $\mathrm{H}_{2}$-induzierten freien Ladungsträger zu einer erhöhten Absorption führen und somit der dominante physikalische Effekt bei der beobachteten, optischen Sensorantwort sind.

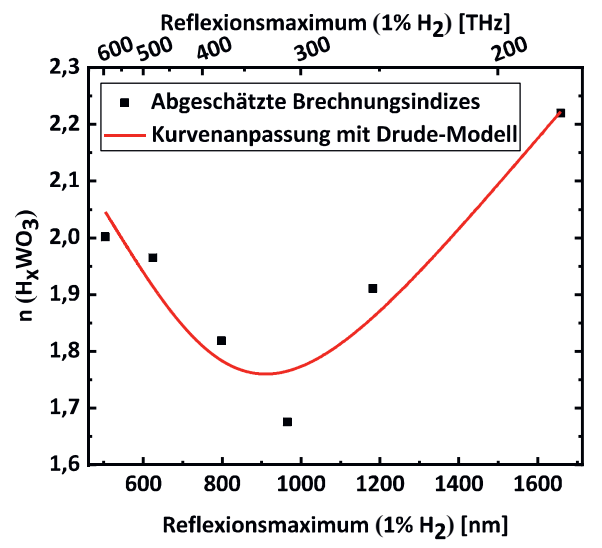

Abb. 4: Abgeschätzte Dispersion des Realteils des Brechungsindexes von $\mathrm{H}_{x} \mathrm{WO}_{3}$ (schwarz) und angepasstes Drude-Modell (rote Kurve).

\section{Zusammenfassung}

Wir haben gezeigt, dass die Absorption durch freie Ladungsträger eine wichtige Verbindung zwischen den mikroskopischen elektronischen und den makroskopischen optischen
Eigenschaften bei der Gasreaktion von halbleitenden Metalloxiden darstellt. Mit einem Modellsystem $\left(\mathrm{WO}_{3} / \mathrm{H}_{2}\right)$ ist es uns gelungen, die Dispersion des Metalloxids unter Gasangebot mit Hilfe einer Reihe von Inversopalen mit unterschiedlicher photonische Bandlücke zu rekonstruieren. Die so erhaltene Dispersionskurve ließ sich schließlich mit Hilfe des DrudeModells anpassen. Dies ist ein starker Hinweis auf die Absorption durch freie Ladungsträger als dominierender Mechanismus für die beobachtete Gasreaktion.

Zusätzlich konnte in der Anwendung mit Hilfe der Dispersionskurve die Sensorantwort um eine Größenordnung erhöht werden.

Ausgehend von diesen Ergebnissen gehen wir davon aus, dass die meisten halbleitenden Metalloxide für die optische Gassensorik mit Hilfe der Inversopalstruktur genutzt werden können. Allerdings muss der spektrale Bereich so gewählt, dass dieser nah genug an einem Bereich starker Dispersion der optischen Konstanten des jeweiligen Metalloxids liegt, um eine hinreichend starke optische Sensorantwort zu erhalten. Für die meisten Metalloxide liegt dieser im IR-Bereich und wird bestimmt durch die Variation der freien Ladungsträgerdichte. ${ }^{[3]}$

\section{Danksagung}

Wir danken dem Bundesministerium für Bildung und Forschung (FKZ: 13N12969) für die finanzielle Unterstützung.

\section{Literaturnachweis}

[1] Mark Fox, Optical properties of solids, 2. Ed, Oxford University Press, New York, USA 2010

[2] M. Ando, T. Kobayashi, M. Haruta, Catal. Today 1997, 36, 135

[3] M. -I. Baraton and L. Merhari, Synth. React. Inorg., Met.-Org., Nano-Met. Chem. 2005, 35, 733

[4] K. Ito and T. Ohgami, Appl. Phys. Lett. 1992, 60, 938

[5] A. Georg, W. Graf, R. Neumann, V. Wittwer, Solid State lonics 2000, 127, 319

[6] D. Nau, A. Seidel, R.B. Orzekowsky, S.-H. Lee, S. Deb, H. Giessen, Opt. Lett. 2010, 35, 3150

[7] Z. Xie, H. Xu, F. Rong, L. Sun, S. Zhang, Z. Gu, Thin Solid Films 2012, 520, 4063

[8] P.J. Shaver, Appl. Phys. Lett. 1967, 11, 255

[9] H. Chen, N. Xu, S. Deng, D.Lu, Z. Li, J. Zhou, J. Chen, Nanotechnology 2007, 18, 205701

[10] S. H. Lee, H. M. Cheong, P. Liu, D. Smith, C. E. Tracy, A. Mascanrenhas, J. R. Pitts, S. K. Deb, J. Appl. Phys. 2000, 88, 3076

[11] S. Okazaki, H. Nakagawa, S. Asakura, Y. Tomiuchi, N. Tsuji, H. Murayama, M. Washiya, Sens. Actuators, B. 2003, 93, 142

[12] C.G. Granqvist, Handbook of Inorganic Electrochromic Materials, Elsevier, Amsterdam, The Netherlands 1995 
[13] S. Amrehn, X. Wu, T. Wagner, ACS Sens. 2018, 3, 191

[14] M. Egen, R. Zentel, Macromol. Chem. Phys 2004, 205, 1479

[15] H. Míguez, C. López, F. Meseguer, A. Blanco, L. Vázquez, R. Mayoral, Appl. Phys. Lett. 1997, 71, 1148

[16] A. Reynolds, F. López-Tejeira, D. Cassagne, F. J. García-Vidal, C. Jouanin, J. SánchezDehesa, Phys. Rev. B. 1999, 60, 11422

[17] R. B. Goldner, P. Norton, K. Wong, G. Foley, E. L. Goldner, G. Seward, R. Chapman, Appl. Phys. Lett. 1985, 47, 536 